\title{
Stem-loop structure preference for site-specific RNA editing by APOBEC3A and APOBEC3G
}

\author{
Shraddha Sharma ${ }^{\text {Corresp., } 1}$, Bora E Baysal ${ }^{\text {Corresp. } 1}$ \\ ${ }^{1}$ Department of Pathology, Roswell Park Cancer Institute, Buffalo, New York, United States \\ Corresponding Authors: Shraddha Sharma, Bora E Baysal \\ Email address: shraddha.sharma@roswellpark.org, Bora.Baysal@roswellpark.org
}

APOBEC3A and APOBEC3G cytidine deaminases inhibit viruses and endogenous retrotransposons. We recently demonstrated the novel cellular C-to-U RNA editing function of APOBEC3A and APOBEC3G. Both enzymes deaminate single-stranded DNAs at multiple TC or CC nucleotide sequences, but edit only a select set of RNAs, often at a single TC or CC nucleotide sequence. To examine the specific site preference for APOBEC3A and -3Gmediated RNA editing, we performed mutagenesis studies of the endogenous cellular RNA substrates of both proteins. We demonstrate that both enzymes prefer RNA substrates that have a predicted stem-loop with the reactive $C$ at the $3^{\prime}$-end of the loop. The size of the loop, the nucleotides immediately 5 ' to the target cytosine and stability of the stem have a major impact on the level of RNA editing. Our findings show that both sequence and secondary structure are preferred for RNA editing by APOBEC3A and -3G, and suggest an explanation for substrate and site-specificity of RNA editing by APOBEC3A and -3G enzymes. 
1 Stem-loop structure preference for site-specific RNA editing by APOBEC3A

2 and APOBEC3G

3 Shraddha Sharma ${ }^{1^{*}}$ and Bora E. Baysal ${ }^{1^{*}}$

$4{ }^{1}$ Department of Pathology, Roswell Park Cancer Institute, Buffalo, New York, 14263, USA

5 *To whom correspondence should be addressed

6 Dr. Bora E. Baysal

$7 \quad$ Email:Bora.Baysal@roswellpark.org

8

9 Correspondence may also be addressed to

10 Dr. Shraddha Sharma

11 Email: shraddha.sharma@roswellpark.org

12

13

14

15

16

17

18

19

20

21 
22 ABSTRACT

23 APOBEC3A and APOBEC3G cytidine deaminases inhibit viruses and endogenous

24 retrotransposons. We recently demonstrated the novel cellular C-to-U RNA editing function of

25 APOBEC3A and APOBEC3G. Both enzymes deaminate single-stranded DNAs at multiple TC or

26 CC nucleotide sequences, but edit only a select set of RNAs, often at a single TC or CC

27 nucleotide sequence. To examine the specific site preference for APOBEC3A and -3G-mediated

28 RNA editing, we performed mutagenesis studies of the endogenous cellular RNA substrates of

29 both proteins. We demonstrate that both enzymes prefer RNA substrates that have a predicted

30 stem-loop with the reactive $\mathrm{C}$ at the 3 '-end of the loop. The size of the loop, the nucleotides

31 immediately $5^{\prime}$ to the target cytosine and stability of the stem have a major impact on the level of

32 RNA editing. Our findings show that both sequence and secondary structure are preferred for

33 RNA editing by APOBEC3A and -3G, and suggest an explanation for substrate and site-

34 specificity of RNA editing by APOBEC3A and -3G enzymes.

35 INTRODUCTION

36 The APOBEC3 (A3) family of cytidine deaminases restricts endogenous retroelements and

37 exogenous viruses and therefore plays an important role in the vertebrate innate immune system

38 [Cullen, 2006; Chiu and Greene, 2008; Harris and Dudley, 2015]. Recent studies also suggest that

39 A3 enzymes help retroviruses escape from drugs and adaptive immune recognition (Monajemi et

40 al., 2012; Grant and Larijani, 2017). The A3 family comprises seven homologous enzymes in

41 primates [Jarmuz et al., 2002; Conticello, 2008; Prohaska et al., 2014] that have either one (A3A,

$42 \mathrm{~A} 3 \mathrm{C}$ and $\mathrm{A} 3 \mathrm{H})$ or two $(\mathrm{A} 3 \mathrm{~B}, \mathrm{~A} 3 \mathrm{D}, \mathrm{A} 3 \mathrm{~F}$ and $\mathrm{A} 3 \mathrm{G})$ zinc $(\mathrm{Zn})$-coordinating catalytic domains with

$43 \mathrm{HX}_{1} \mathrm{EX}_{23-24} \mathrm{CX}_{2-4} \mathrm{C}$ motifs ( $\mathrm{X}$ is any amino acid). The histidine and cysteine residues coordinate

$44 \mathrm{Zn}^{2+}$ [Betts et al., 1994], and the glutamic acid residue may function as a proton shuttle during the

45 deaminase reaction [Betts et al., 1994]. 
47 However, prior structural and biochemical studies have focused on the interaction of A3 enzymes

48 and ssDNA oligonucleotides since C-to-U $(\mathrm{C}>\mathrm{U})$ deamination has been demonstrated in ssDNA

49 exclusively. The A3 family members prefer a thymine immediately 5 ' to the target C, except

50 APOBEC3G $(\mathrm{A} 3 \mathrm{G})$, which prefers a cytosine at the 5' position in their ssDNA substrates

51 [Refsland and Harris, 2013 and references therein]. A study by Mitra et al., reported that ssRNA

52 is not a substrate for A3A since ssRNA binds to A3A weakly as compared to ssDNA and A3A-

53 mediated ssRNA deamination was not detected [Mitra et al., 2014]. A3G has been shown to bind

54 to both ssDNA and ssRNA with similar affinities [Iwatani et al., 2006]. While A3G deaminates

55 ssDNA, no deamination was detected in ssRNA [Iwatani et al., 2006]

APOBEC3A (A3A) is highly expressed in monocytes and macrophages and its expression is upregulated on treatment with interferon- $\alpha$ [Chen et al, 2006; Peng et al., 2007; Koning et al., 2009]. We recently demonstrated the novel RNA editing function of A3A in monocytes and monocyte-derived macrophages [Sharma et al., 2015]. A3A induces site-specific RNA editing in mRNAs from hundreds of genes in response to low-oxygen (hypoxia) and interferon type 1 (IFN1) treatment. Of these edited transcripts, 128 out of 211 and 93 out of 116 edited sites are in the coding exons in monocytes and macrophages, respectively [Sharma et al., 2015]. On transiently expressing A3A in HEK293T cells, mRNAs of thousands of genes undergo site-specific editing

[Sharma et al., 2017]. Furthermore, we demonstrated site-specific editing of ssRNA with purified recombinant $\mathrm{A} 3 \mathrm{~A}$ in vitro, whereas DNA editing is non-specific and occurs at multiple T므 nucleotides (edited C underlined) [Sharma et al., 2015]. More recently, we have identified the RNA editing function of a second member of the A3 family- the two-domain cytidine deaminase and an anti-HIV-1 restriction factor A3G by transient expression in HEK293T cells [Sharma et al., 2016]. Interestingly, computational analysis revealed that the edited targets in $>70 \%$ of $\mathrm{A} 3 \mathrm{~A}$ 
71 palindromic sequences [Sharma et al., 2015; Sharma et al., 2017]. In the case of A3G, $98 \%$ of

72 the edited targets are flanked by inverted repeats in 293T cells [Sharma et al., 2016].These

73 bioinformatic observations suggested that RNAs with predicted stem-loop structures may be

74 preferentially targeted for RNA editing by A3A and A3G [Sharma et al., 2015; Sharma et al.,

75 2016; Sharma et al., 2017]. However, the underlying mechanism for this preference is not clear.

76 To test the hypothesis that RNA stem-loop structure is important for RNA editing by A3A and

77 A3G, we generated a panel of RNA mutants and examined the features of endogenous substrates

78 of $\mathrm{A} 3 \mathrm{~A}$ and $\mathrm{A} 3 \mathrm{G}$ required for RNA editing. Here we experimentally demonstrate for the first time

79 the preference for a stem-loop structure for site-specific A3A and A3G-mediated RNA editing.

80

81 MATERIAL AND METHODS

82 Cell culture, plasmids and transfection

83 Cell cultures of primary monocyte-enriched PBMCs, exposure to hypoxia (1\% oxygen) and

84 interferon type 1 were performed as previously described [Sharma et al., 2015].

85 Plasmid constructs for expression of human A3A cDNA, for the generation of C-terminal Myc-

86 DDK-tagged A3A and A3G, pcDNA 3.1(+) vector (used as an empty vector control) were

87 obtained from sources mentioned in [Sharma et al., 2015; Sharma et al., 2016].

88 The TLA-HEK293T human embryonic kidney cells (293T cells) (Open Biosystems) were

89 transfected with plasmid DNA using the jetPRIME (Polyplus-transfection) reagent as per the

90 manufacturer's instructions. The transfection efficiency was $60 \%-80 \%$ as assessed by fluorescent

91 microscopy of cells that were transfected with the pLemiR plasmid (Open Biosystems) for

92 expression of a red fluorescent protein. Cells were harvested 2 days following transfection.

\section{Purification of recombinant A3A proteins}


94 The WT A3A was purified as described in [Sharma et al., 2015]. Briefly, Rosetta 2(DE3)pLysS

95 E. coli (EMD Millipore) transformed with a bacterial expression construct for C-terminal His $6^{-}$

96 tagged WT A3A was grown in Luria broth at $37^{\circ} \mathrm{C}$. The cells were induced for expression of the

97 recombinant protein with $0.3 \mathrm{mM}$ isopropyl $\beta$-D-1-thiogalactopyranoside and cultured overnight

98 at $18{ }^{\circ} \mathrm{C}$. A3A protein was purified from the lysates by affinity chromatography using the Ni-

99 NTA His bind Resin (EMD Millipore). The concentrated protein was stored in $25 \mathrm{mM}$ Tris ( $\mathrm{pH}$

1008.0 ) with $50 \mathrm{mM} \mathrm{NaCl}, 1 \mathrm{mM}$ DTT, $5 \%$ v/v glycerol and $0.02 \% \mathrm{w} / \mathrm{v}$ sodium azide at $-80{ }^{\circ} \mathrm{C}$.

\section{Predicting RNA secondary structures}

10218 nucleotides (with 7 nucleotides flanking on each side of the tetra-loop sequence) of WT

103 SDHB, TMEM109 and APP RNAs were folded using the Mfold nucleic acid folding program

104 [Zuker, 2003].18 nucleotides of WT PRPSAP2 RNA were folded using both mfold and RNAfold

105 2.3.2 [Zuker, 2003; Lorenz et al., 2011]. No optional parameters were used. A single structure

106 along with the minimum free energy value for the structure was obtained for the selected RNAs

107 and is represented in Supplementary Fig. 1.

108 RNA mutagenesis and RNA editing assays

109 The DNA templates for generating WT and mutant SDHB (except M1, M6, M7), TMEM109 and

110 APP RNAs were amplified using oligonucleotide primers listed in Supplementary Table 1. M1,

111 M6 and M7 SDHB RNAs were generated from the $1.1 \mathrm{~kb}$ complete SDHB ORF encoding

112 plasmid (RC203182, Origene) following site-directed mutagenesis and XhoI linearization of the

113 plasmid DNA. Sanger sequencing was performed on all DNA templates to confirm the desired

114 mutations, which were then in vitro transcribed to generate RNAs using reagents and methods

115 provided with the MEGAscript or MEGAshortscript T7 Transcription Kit (Life Technologies).

116 RNAs isolated from the transcription reaction were treated with DNAse I (Thermo Fisher) and

117 their integrity was verified by electrophoresis on an agarose gel. 
118 In vitro RNA-editing assay with purified APOBEC3A contained 1-10 $\mu \mathrm{M}$ APOBEC3A, 50 pg of

119 synthetic RNAs, $10 \mathrm{mM}$ Tris (pH 8.0), $50 \mathrm{mM} \mathrm{KCl}$ and $10 \mu \mathrm{M} \mathrm{ZnCl}_{2}$. The reactions were

120 incubated for 2 hours at $37^{\circ} \mathrm{C}$. RNA was purified from the reactions using TRIzol (Life

121 Technologies) as per the manufacturer's instructions and reverse transcribed to generate cDNAs

122 as described previously [Sharma et al., 2015]. The 136C $>\mathrm{U}$ editing of the WT and certain $S D H B$

123 RNA mutants (M1, M3-M7) was assessed by allele-specific AS-RT-qPCR as described

124 previously [Sharma et al., 2015; Baysal et al., 2013], whereas RNA editing levels for remainder

125 of the mutant RNAs along with the WT controls were determined by Sanger sequencing, using

126 the primers listed in Supplementary Table 1, because these mutants could not be amplified by AS-

127 RT-qPCR reverse primers (Supplementary Table 2, Supplementary Data). We have previously

128 shown (Sharma et al., 2015, Supplementary Fig. 8) a strong positive correlation (r=0.94) between

$129 S D H B$ 136C $>$ U RNA editing level measurements obtained by AS-RT-qPCR and Sanger

130 sequencing, although estimates obtained by Sanger sequencing were somewhat higher (slope of

131 correlation=0.71). Thus, it is possible that true editing levels in SDHB constructs M2, M8, M9

132 and M10, which were estimated by Sanger sequencing (Supplementary Data), might be actually

133 lower (by approximately 30\%).

134 Since in vitro RNA editing by A3G has not yet been demonstrated, to examine the impact of

135 RNA mutations in the A3G substrate PRPSAP2 on RNA editing, we co-transfected mutated

136 PRPSAP2 expression plasmid with A3G expression plasmid in 293T cells. The mutations were

137 performed by site-directed mutagenesis (New England Biolabs) in the PRPSAP2 expression

138 plasmid (clone ID Ohu59963, RefSeq accession XM_011523960; GenScript). Total RNA was

139 isolated and RT-PCR was performed using a PRPSAP2-specific forward primer and a vector

140 specific reverse primer complementary to the DDK tag sequence (Supplementary Table 1). These

141 primers specifically amplified the plasmid derived PRPSAP2 transcripts but not the endogenously 
142 expressed transcripts, allowing us to directly examine the impact of RNA mutations on A3G-

143 mediated RNA editing.

\section{Estimation of RNA editing levels by Sanger sequencing}

145 Sequencing primers (Integrated DNA Technologies) for the WT and mutant cDNAs generated

146 from RNAs are listed and underlined in Supplementary Table 1. The PCR products were

147 examined by agarose gel electrophoresis to verify their size and then sequenced on the $3130 \mathrm{xL}$

148 Genetic Analyzer (Life Technologies) at the RPCI genomic core facility as described in Sharma

149 et al. 2016. The major and minor chromatogram peak heights at putative edited nucleotides were

150 quantified with Sequencher 5.0/5.1 software (Gene Codes, Ann Arbor, MI) in order to calculate

151 the editing level for the position (Supplementary Data). Since the software identifies a minor

152 peak only if its height is at least $5 \%$ that of the major peak's, we have considered $0.048[=5 /(100+$

153 5)] as the detection threshold (Sharma et al. 2016; Supplementary Data).

155 RESULTS

157 editing

158 Previous studies have shown that A3A-mediated DNA deamination of synthetic

159 oligonucleotides occurs non-specifically at TC dinucleotides [Chen et al., 2006, Shinohara et al.,

160 2012; Sharma et al., 2015; Chan et al., 2015]. However, A3A-mediated cellular ssRNA editing is

161 site-specific, and bioinformatic analyses predicted that approximately $70 \%$ of the edited Cs in

162 A3A's RNA substrates are located within secondary structures [Sharma et al., 2015]. The most

163 common secondary structure is predicted to be comprised of a CAU $\underline{\mathrm{C}}$ tetra-loop flanked by an

164 average of three palindromic nucleotides [Sharma et al., 2015]. Similarly, bioinformatics analyses

165 predicted that $\sim 98 \%$ of the edited Cs in A3G RNA substrates are located within secondary 
166 structures; the most common structure comprising of $\mathrm{CNC} \underline{\mathrm{C}}$ ( $\mathrm{N}$ is any nucleotide) flanked by an

167 average of four palindromic nucleotides [Sharma et al., 2016]. Separately, while validating edited

168 sites in primary monocytes by Sanger sequence analysis, we observed that a silent A/G single

169 nucleotide polymorphism (SNP) in the A3A substrate C1QA mRNA (rs172378) markedly

170 increased $\underline{\mathrm{C}}>\mathrm{U}$ RNA editing three nucleotides upstream of the polymorphism (Fig. 1A). The $A>G$

171 change in the CIQA mRNA (rs172378) is predicted to increase the stem length and subsequently

172 the stem stability of a putative stem-loop structure, resulting in increased RNA editing. While the

173 CCCCCUCGG(a/a) (expressed SNP variation in lower case) sequence shows $11 \%$ and $21 \%$

174 editing in 2 donors, CCCCCUCGG(a/g) increased the average editing to $40 \%$ when monocyte-

175 enriched peripheral blood mononuclear cells (MEPs) were exposed to hypoxia/IFN-1 (Fig. 1A).

176 Although the estimated RNA editing level may have been affected by background noise (in donor

1772 and 3 hypoxia/IFN-1 samples), visual inspection of the chromatograms clearly show that the

178 heterozygous donor 1 cellular RNAs are edited at a higher level compared to homozygous donor

1792 and 3 cellular RNAs.

180 We thus hypothesized that stem-loop RNAs are preferred substrates for editing by

181 APOBEC3A and -3G proteins. We selected three site-specifically edited A3A mRNA substrates-

$182 S D H B\left(N M \_003000: c .136 C>U, R 46 X\right), A P P\left(N M \_001204302: c .1546 C>U, R 516 C\right)$,

183 TMEM109 (NM_024092: c.109C>U, R37X) [Sharma et al., 2015; Sharma et al., 2017] and one

184 such A3G substrate, PRPSAP2 (NM_001243941: c.664C>U, R222W) [Sharma et al., 2016] for

185 further analysis. On analysis of 18 nucleotides of RNA sequence containing the target $\underline{\mathrm{C}}$ by the

186 mfold [Zuker, 2003] or RNAfold [Lorenz et al., 2011] nucleic acid folding prediction programs,

187 secondary structures with $\triangle \mathrm{G}$ values between -5 to $-6 \mathrm{kcal} / \mathrm{mol}$ are predicted for $S D H B, A P P$ and

188 PRPSAP2 RNAs (Supplementary Fig. 1). The predicted secondary structure for these RNAs is a

189 tetra-loop with the edited $\underline{\mathrm{C}}$ at the $3^{\prime}$ end of the loop flanked by a stem containing 3-5 base pairs

190 (bp). TMEM109 is predicted to form a hepta-loop with a four bp stem and a $\Delta \mathrm{G}$ value of -1.7 
$191 \mathrm{kcal} / \mathrm{mol}$. To test the importance of stem-loop structures for A3A and A3G-mediated RNA

192 editing, we created various mutations (see methods) in the putative loop and stem regions of A3A

193 substrates $S D H B, A P P$ and TMEM109 and the A3G substrate PRPSAP2 (Fig. 1B, C and D) and

194 assessed their editing levels. SDHB, APP, TMEM109 RNAs show $\sim 83 \%, 24 \%, 51 \%$ site-specific

195 editing in an in vitro system, respectively and PRPSAP2 shows $44 \%$ RNA editing in a cell based

196 system. The RNA editing levels were analysed by AS-RT-qPCR for the SDHB mutants, except

197 those which did not have a reverse primer compatible for AS-RT-qPCR analysis of RNA editing

198 (see methods; Supplementary Table 2). The remainder of the SDHB, APP, TMEMI09 and

199 PRPSAP2 mutants were analysed by Sanger sequencing (Supplementary Data). In either method

200 for assessing RNA editing levels, WT RNA substrates were used as a positive control. For

201 convenience in data interpretation, RNA editing of WT substrates is set to $100 \%$ and that of

202 mutant RNAs is reported as a fraction of that observed with the WT substrates.

203 We tested the importance of the -1 nucleotide (nt) (immediately $5^{\prime}$ to $\underline{\mathrm{C}}$ ) in $\mathrm{A} 3 \mathrm{~A}$ and $\mathrm{A} 3 \mathrm{G}$

204 substrates. $\mathrm{C}>\mathrm{U}$ editing sites are most commonly present within a CCAU $\underline{\mathrm{CG}}$ sequence motif in

205 ssRNA A3A substrates [Sharma et al., 2015]. Changing the $-1 \mathrm{U}$ to $\mathrm{C},(\mathrm{U} \underline{\mathrm{C}}>\mathrm{C} \underline{\mathrm{C}})$ in the predicted

206 loop region of the $S D H B$ RNA (Fig. 1B, M1), markedly reduced A3A-mediated RNA editing

207 from the normalized value of $100 \%$ to $19 \%$. Unlike most A3A substrates, which prefer U at -1

208 position, in $A P P$ RNA the -1 nt is occupied by C. Interestingly, substituting $\mathrm{C}$ at -1 with $\mathrm{U}$ in

$209 A P P$ RNA (C $\underline{\mathrm{C}}>\mathrm{U} \underline{\mathrm{C}}$ ), increased editing to 364\% (Fig. 1C, M1A). The majority of $\mathrm{C}>\mathrm{U}$ editing

210 sites in $\mathrm{A} 3 \mathrm{G}$ substrates are present within a $\mathrm{CNC} \underline{\mathrm{C}}[\mathrm{A} / \mathrm{G}]$ sequence and therefore prefer $\mathrm{C}$ at -1

211 position [Sharma et al., 2016]. Changing -1 nt to $\mathrm{G}(\mathrm{C} \underline{\mathrm{C}}>\mathrm{G} \underline{\mathrm{C}})$ in the A3G substrate PRPSAP2

212 RNA loop markedly reduced RNA editing to $15 \%$ as compared to WT (Fig. 1D, M1P). These

213 results suggest a preference for $\mathrm{U}$ and $\mathrm{C}$ at the -1 position in the loop regions of $\mathrm{A} 3 \mathrm{~A}$ and $\mathrm{A} 3 \mathrm{G}$

214 substrates, respectively. 

region of the A3A RNA substrate, $S D H B$. As mentioned above, our computational analysis

217 predicts that the edited $\underline{\mathrm{C}}$ is generally located at the $3^{\prime}$ end of the tetra-loop [Sharma et al., 2015;

218 Sharma et al., 2016; Sharma et al., 2017]. Changing the position of edited $\underline{\mathrm{C}}$ one nucleotide

219 upstream within the loop in $S D H B$ RNA, while maintaining $\mathrm{U}$ at -1 position, greatly reduced

220 RNA editing to $10 \%$ (Fig. 1B, M2). This result suggests that position of the reactive $\underline{\mathrm{C}}$ within the 221 loop is critical for RNA editing.

The majority of known A3A and A3G RNA substrates are predicted to form a tetra-loop structure [Sharma et al., 2015; Sharma et al., 2016; Sharma et al., 2017]. To test whether the size of the loop plays a role in RNA editing, we created substitutions that increase or decrease the predicted loop size in $S D H B$ and PRPSAP2 RNAs (Fig. 1B, M3 and M4; Fig. 1D, M2P and M3P). Increasing from a tetra-loop to a penta-loop (Fig. 1B, M3) reduced RNA editing to 10\% in the $S D H B$ RNA, and decreasing the size to a tri-loop (Fig. 1B, M4) diminished editing to $60 \%$ as compared to the WT SDHB RNA. Changing the size of the loop (penta- or tri-loop) of the PRPSAP2 RNA abolished A3G-mediated RNA editing (Fig. 1D, M2P and M3P). These results suggest that a larger loop is detrimental to both A3A and A3G-mediated RNA editing, whereas

231 reducing the size of the loop to three nucleotides may be tolerated better. stem are determinants of RNA editing. We weakened or disrupted the predicted stem by

234 decreasing the number of complementary base pairs in SDHB and PRPSAP2 RNAs (Fig. 1B, M5, M6 and M7; Fig. 1D, M4P and M5P). All of these changes reduced SDHB RNA editing 5-10 fold to $16 \%, 12 \%$ and 10\%, respectively (Fig. 1B M5, M6 and M7) and abolished A3G-mediated RNA editing of PRPSAP2 (Fig. 1D, M4P and M5P). Further, altering (inverting/swapping) the sequence of the stem while maintaining base-pairing of the $S D H B$ RNA (Fig. 1B, M8 and M9) 
240 observations suggest that both sequence and stability of the RNA structure are important for

241 optimum RNA editing.

242 Usually the +1 position (with regard to $\underline{\mathrm{C}}$ ) in $\mathrm{A} 3 \mathrm{~A}$ substrates is occupied by a $\mathrm{G}$ base-

243 paired with $\mathrm{C}$ or in some cases A, which was substituted with A in M8 (37\% editing) and C in M9

244 (17\% editing) (Fig. 1B). Hence, to test the importance of $\mathrm{G}$ at +1 position, we created another

245 mutant that retained the first base pair of the predicted stem ( $\mathrm{G}$ at +1$)$ as WT SDHB, but

246 remainder of the stem sequence and structure was similar to the M9 SDHB mutant (Fig. 1B, M9

247 and M10). Changing $\mathrm{C}$ at +1 position in the $\mathrm{M} 9$ mutant to $\mathrm{G}$ in the M10 mutant (Fig. 1B)

248 increased A3A-mediated RNA editing from $\sim 17 \%$ to $130 \%$, respectively (Fig. 1B). These results

249 suggest that the structure/stability rather than sequence of the predicted stem, other than $G$ at +1

250 position determines the level of RNA editing.

251 To further examine the importance of the stability and structure of the stem, we analyzed

252 the A3G RNA substrate, PRPSAP2 and the A3A substrate TMEM109. Interestingly, weakening

253 the putative stem by substituting two G-C base pairs with A-U base pairs in PRPSAP2 RNA only

254 affected RNA editing slightly (80\%) (Fig. 1D, M6P). As mentioned above, disrupting the

255 predicted stem structure abolished RNA editing in PRPSAP2 (Fig. 1D, M5P). However, on

256 swapping the $5^{\prime}$ and 3 ' sequence while maintaining the stem complementarity as well as the first

257 C-G base pair, increased RNA editing to $180 \%$ as compared to WT PRPSAP2 (Fig. 1D, M7P).

258 Similarly, when we compare the SDHB RNA mutants M6 and M10 (Fig. 1B), restoring the stem

259 stability and structure, while maintaining $\mathrm{G}$ at +1 position increased RNA editing from $12 \%$ to

$260130 \%$. These results provide further evidence that stem stability and $\mathrm{G}$ at +1 position, rather than

261 nucleotide sequence in the remainder of the predicted stem region determine the level of RNA

262 editing.

263 As mentioned above, for the A3A substrate TMEM109, the mfold program predicts a

264 hepta-loop flanked by a four bp stem (Supplementary Fig. 1). However, if the unpaired 
265 adenosine in the hepta-loop region bulges out then we predict WT TMEM109 RNA to form a

266 tetra-loop with $\underline{\mathrm{C}}$ at the $3^{\prime}$ end of the loop, $\mathrm{G}$ at +1 position base paired with $\mathrm{C}$ and a 5 bp long

267 stem (Fig. 1C). To test the effect of perfect stem complementarity on TMEM109 RNA editing

268 level, we deleted the unpaired adenosine (Fig. 1C, M1T). Unlike for WT TMEM109 $(\triangle \mathrm{G}=-1.7$

$269 \mathrm{kcal} / \mathrm{mol}$ ), the mfold program predicts a $\Delta \mathrm{G}$ value of $-5.2 \mathrm{kcal} / \mathrm{mol}$ for $T M E M 109 \mathrm{M} 1 \mathrm{~T}$ structure

270 (Supplementary Fig. 1), suggesting an increase in secondary structure stability. Deletion of the

271 unpaired adenosine to obtain perfect stem complementarity resulted in an increase in the RNA

272 editing level of TMEM109 from 100\% to 122\% (Fig. 1C, M1T).

273 Taken together, our results show that for site-specific RNA editing, A3A and A3G prefer a

274 stem-loop secondary structure, with $\underline{\mathrm{C}}$ at the end of the tetra-loop as well as specific nucleotides

275 at $5^{\prime}$ and $3^{\prime}$ positions immediate to the reactive $\underline{\mathrm{C}}$, and suggests that the sequence of the predicted

276 stem other than at +1 position is not as important as the stability of base pairing.

277

278 DISCUSSION

279 Most of the structural and biochemical studies of A3A and A3G thus far have focused on

280 ssDNA substrate binding and the mechanism of catalysis. Moreover, it has been suggested that

281 RNA is not a substrate for A3A and A3G [Iwatani et al., 2006; Mitra et al., 2014]. This is

282 primarily because prior studies have shown DNA editing whereas RNA editing by the APOBEC3

283 enzymes was not observed until we demonstrated the RNA editing function of A3A and A3G

284 recently [Sharma et al., 2015; Sharma et al., 2016; Sharma et al., 2017]. The observation that

285 RNA editing is site-specific with edited NNNC flanked by inverted repeats, whereas DNA editing

286 occurs non-specifically at dinucleotide $[\mathrm{T} / \mathrm{C}] \underline{\mathrm{C}}$ sequences motivated us to investigate the RNA

287 secondary structure preference for A3A and A3G. Here, we show that stem-loop structures, with

288 the reactive $\underline{\mathrm{C}}$ contained in the loop, are preferred substrates for site-specific $\mathrm{A} 3 \mathrm{~A}$ and $\mathrm{A} 3 \mathrm{G}-$

289 mediated RNA editing (Fig. 1). 
290 Our results suggest that the determinants of RNA editing lie within the predicted loop of

291 the stem loop structure, the +1 nucleotide in the stem, while the level of editing may be

292 determined by the stem stability. Changing T두 C $\underline{C}$ in the SDHB RNA (A3A substrate) and

293 changing $\mathrm{C} \underline{\mathrm{C}}>\mathrm{G} \underline{\mathrm{C}}$ in PRPSAP2 RNA (A3G substrate) markedly reduces or abolishes RNA

294 editing by these enzymes respectively, thus highlighting the importance of the $-1 \mathrm{nt}$ in the loop

295 (Fig. 1B, M1 and Fig. 1D, M1P). Another important feature is the +1 nucleotide (G) located in

296 the putative stem common to all substrates of A3A and A3G examined here. Any substitution of

$297 \mathrm{G}$ at the +1 position in these substrates markedly reduces RNA editing (Fig. 1B, M8, M9). In

298 contrast to a predicted tetra-loop or a tri-loop, a predicted penta-loop RNA shows poor editing by

299 both A3A and A3G (Fig. 1B, M3 and Fig. 1D, M2P). This may be because the catalytic site of

300 these proteins is not 'open' or flexible enough to accommodate the larger RNA loop or because $\underline{\mathrm{C}}$

301 is not present at the end of the loop in these mutants. The level of RNA editing by A3A and A3G

302 in SDHB and PRPSAP2 RNAs, respectively increases when compared to WT when the predicted

303 stem sequence is altered while retaining the first base pair and the stem stability (Fig. 1B, M10

304 and Fig. 1D, M7P) or if we increase the stem stability of the A3A substrate TMEM109 RNA by

305 deleting the unpaired adenosine (Fig. 1C, M1T). These mutations may result in a more

306 energetically favourable secondary structure for RNA editing or may result in a better 'fit' and

307 interaction of the bases with the catalytic and surrounding residues.

308 Secondary structures of RNAs have been previously shown to aid in site-specific editing by

309 adenosine deaminases in both prokaryotes and eukaryotes. The adenosine deaminases, ADARs,

310 act on double stranded RNAs (dsRNAs) to convert adenosine to inosine. Secondary structure in

311 the form of internal loops, bulges and mismatches in the dsRNAs dictate site-specificity in these

312 enzymes resulting in the editing of a few adenosines as compared with long ( $>100 \mathrm{bp})$ dsRNA

313 substrates, in which more than half of the adenosines are edited [Lehmann and Bass, 1999; Bass,

314 2002; Nishikura, 2016; Deffit and Hundley, 2016 ]. The site selectivity in the glutamate receptor 
315 GRIA2, catalyzed by ADAR2, requires a stem structure that is formed between the exonic

316 sequence containing the target $\underline{\mathrm{A}}$ and a downstream intronic complementary sequence, resulting

317 in $>99 \%$ editing efficiency [Higuchi et al., 1993]. Although ADARs prefer $U$ at -1 and $G$ at +1

318 position relative to the edited $\mathrm{A}$, there is no strict sequence requirement for $\mathrm{A}>\mathrm{I}$ editing [Lehmann

319 and Bass, 2000; Nishikura, 2016]. Also, the mechanism which determines the level of A $>$ I RNA

320 editing is not yet clear [Nishikura, 2016].

321 A distant relative of APOBECs, the prokaryotic adenosine deaminase TadA (Adenosine

322 deaminase acting on tRNA or ADAT) has the active site characteristic of the cytidine deaminases

323 and its mechanism of reaction is analogous to that of cytidine deaminases [Carter, 1995; Losey et

324 al., 2006]. TadA deaminates adenosine to inosine at the wobble position $\left(\mathrm{A}^{34}\right)$ of the tRNA ${ }^{\mathrm{Arg} 2}$

325 anticodon stem-loop and involves an induced fit of the RNA stem-loop into an inflexible protein

326 cleft [Losey et al., 2006]. Site-specific editing by TadA in the anticodon stem loop is achieved via

327 its interactions with the loop and the single proximal base-pair of the stem, while the remainder

328 of the stem participates in non-specific interactions with the protein, and the reactive adenosine

329 lies within the deepest pocket on the enzyme [Losey et al., 2006]. Further, mutagenesis studies of

330 the RRNA $^{\text {Arg2 }}$ anti-codon stem-loop suggested the importance of the $-1 \mathrm{nt}$, the size of the loop and

331 structure of the stem as determinants of editing by TadA [Wolf et al., 2002]. Another example for

332 secondary structure requirement for cytidine deamination is the Activation-induced cytidine

333 deaminase (AID), which edits C nucleotides located within transcription bubbles or stem-loop

334 structures in ssDNAs, independent of their sequence (Larijani et al., 2007). Recently, the crystal

335 structure of A3A in complex with ssDNA 15-mer shows the DNA oligonucleotide adopting a bent

336 conformation with $\underline{\mathrm{C}}$ inserted in the active site of A3A [Kouno et al., 2017]. A crystal structure of

337 WT A3A/A3G in complex with its ssRNA substrate is crucial to understand the mechanism of

338 protein-RNA interaction and catalysis. 
340 on the level of RNA editing. The $\mathrm{G}$ allele of a common $\mathrm{A} / \mathrm{G}$ synonymous $\mathrm{SNP}$ in $C 1 Q A$

341 (rs172378) has been previously linked to an increased risk of disease severity and nephritis in

342 systemic lupus erythematosus [Namjou, 2009; Radanova et al., 2015]. We observed that this SNP

343 increases the level of site-specific C $>$ U RNA editing three nucleotides upstream of the

344 polymorphism in primary monocytes exposed to hypoxia and interferons (Fig. 1A). RNA editing

345 levels are $11 \%$ and $21 \%$ in two A/A homozygous donors but are increased to $40 \%$ in an $\mathrm{A} / \mathrm{G}$

346 heterozygous donor (Fig. 1A). Although C1QA RNA editing at this site does not change the

347 amino acid (CUC>CUU, both coding for leucine), our findings provide evidence that the $\mathrm{G}$ allele

348 of rs172378 may alter the secondary structure of mRNA to favor a stronger stem and thereby

349 increase the RNA editing level. This alteration in the predicted stem-loop structure may in turn

350 affect mRNA stability, turnover or translatability [Nackley et al., 2006]. Furthermore, it is

351 conceivable that certain synonymous SNPs could create protein diversity by regulating the level

352 of RNA editing. Few examples from our mutagenesis studies include substitutions in the $S D H B$

353 DNA template (Fig. 1B, M1 and M3), where we changed $-1 \mathrm{~T}>\mathrm{C}(\mathrm{Y} 45 \mathrm{Y})$ and $-4 \mathrm{C}>\mathrm{T}$ (I44I).

354 Although these mutations are synonymous, they markedly reduce the level of c.136드 $>$ RNA

355 editing, which causes R46X alteration in SDHB RNA. Similarly, on making synonymous

356 substitutions in the A3G substrate PRPSAP2 by changing $-1 \mathrm{C}>\mathrm{G}(\mathrm{CCc} \underline{\mathrm{C}}>\mathrm{CCgC}$; $\mathrm{P} 267 \mathrm{P})$ and

$357-4 \mathrm{C}>\mathrm{G}(\mathrm{GCcCCCC}>\mathrm{GCgCCC} \underline{\mathrm{C}}$; A266A) (mutated residue in lower case) (Fig. 1D, M1P and

358 M2P, respectively), there is a drastic reduction in RNA editing ( $\underline{\mathrm{CGG}}>\mathrm{UGG}$; R268W) that causes

359 a missense alteration in PRPSAP2. Mutations in the APP gene have been linked to Alzheimer's

360 disease. When we change $-1 \mathrm{C}>\mathrm{T}(\mathrm{CT} \underline{\mathrm{C} G U}>\mathrm{CTt} \underline{\mathrm{CGU}}$; L515L), this synonymous mutation

361 increases the editing level of the missense RNA alteration ( $\underline{\mathrm{CGU}}>\mathrm{UGU}$; R516C) by 264\% (Fig.

362 1C, M1A). Thus, synonymous SNPs in the vicinity of the target $\underline{\mathrm{C}}$ could alter expression of the

363 translated product by regulating the levels of site-specific recoding $C>U$ RNA editing. 


\section{CONCLUSIONS}

367 RNA editing is a mechanism to diversify information encoded by a gene and of regulation

368 of gene expression. Our work provides the first experimental information on how stem-loop

369 structures of endogenous RNA substrates may be preferred for site-specific editing mediated by

370 A3A and A3G cytidine deaminases that are highly expressed in innate immune cells. These

371 enzymes have hundreds of substrates and a single synonymous mutation altering the secondary

372 structure in the substrate RNA could have consequences on the resulting protein product. It is

373 possible that other APOBEC3 enzymes may prefer stem-loop structures, pending the discovery of

374 their RNA editing function. Thus, this study provides the basis for future structural and functional

375 studies.

376

\section{ACKNOWLEDGEMENT}

378 We thank Paul Gollnick for critical reading of the manuscript and for his suggestions. We thank

379 Sally M. Enriquez for purification of the C-terminal his tagged WT A3A protein.

\section{REFERENCES}

Bass BL. 2002. RNA editing by adenosine deaminases that act on RNA. Annu Rev Biochem

383 71:817-846. 10.1146/annurev.biochem.71.110601.135501 
384 Baysal BE, De Jong K, Liu B, Wang J, Patnaik SK, Wallace PK, and Taggart RT. 2013. Hypoxia385 inducible C-to-U coding RNA editing downregulates SDHB in monocytes. PeerJ 1:e152. $386 \quad 10.7717 /$ peerj.152

387 Betts L, Xiang S, Short SA, Wolfenden R, and Carter CW, Jr. 1994. Cytidine deaminase. The 2.3

388 A crystal structure of an enzyme: transition-state analog complex. J Mol Biol 235:635-656.

389 10.1006/jmbi.1994.1018

390 Carter CW, Jr. 1995. The nucleoside deaminases for cytidine and adenosine: structure, transition 391 state stabilization, mechanism, and evolution. Biochimie 77:92-98.

392 Chan K, Roberts SA, Klimczak LJ, Sterling JF, Saini N, Malc EP, Kim J, Kwiatkowski DJ, Fargo

393 DC, Mieczkowski PA, Getz G, and Gordenin DA. 2015. An APOBEC3A hypermutation

394 signature is distinguishable from the signature of background mutagenesis by APOBEC3B in

395 human cancers. Nat Genet 47:1067-1072. 10.1038/ng.3378

396 Chen H, Lilley CE, Yu Q, Lee DV, Chou J, Narvaiza I, Landau NR, and Weitzman MD. 2006.

397 APOBEC3A is a potent inhibitor of adeno-associated virus and retrotransposons. Curr Biol 398 16:480-485. 10.1016/j.cub.2006.01.031

399 Chiu YL, and Greene WC. 2008. The APOBEC3 cytidine deaminases: an innate defensive 400 network opposing exogenous retroviruses and endogenous retroelements. Annu Rev Immunol $401 \quad 26: 317-353.10 .1146 /$ annurev.immunol.26.021607.090350

402 Conticello SG. 2008. The AID/APOBEC family of nucleic acid mutators. Genome Biol 9:229.

$403 \quad 10.1186 / \mathrm{gb}-2008-9-6-229$

404 Cullen BR. 2006. Role and mechanism of action of the APOBEC3 family of antiretroviral 405 resistance factors. J Virol 80:1067-1076. 10.1128/JVI.80.3.1067-1076.2006 
406 Deffit SN, and Hundley HA. 2016. To edit or not to edit: regulation of ADAR editing specificity 407 and efficiency. Wiley Interdiscip Rev RNA 7:113-127. 10.1002/wrna.1319

408 Grant M, and Larijani M. 2017. Evasion of adaptive immunity by HIV through the action of host

409 APOBEC3G/F enzymes. AIDS Res Ther 14:44. 10.1186/s12981-017-0173-8

410 Harris RS, and Dudley JP. 2015. APOBECs and virus restriction. Virology 479-480:131-145.

$411 \quad 10.1016 /$ j.virol.2015.03.012

412 Higuchi M, Single FN, Kohler M, Sommer B, Sprengel R, and Seeburg PH. 1993. RNA editing of

413 AMPA receptor subunit GluR-B: a base-paired intron-exon structure determines position and 414 efficiency. Cell 75:1361-1370.

415 Iwatani Y, Takeuchi H, Strebel K, and Levin JG. 2006. Biochemical activities of highly purified, 416 catalytically active human APOBEC3G: correlation with antiviral effect. $J$ Virol 80:5992-6002.

417 10.1128/JVI.02680-05

418 Jarmuz A, Chester A, Bayliss J, Gisbourne J, Dunham I, Scott J, and Navaratnam N. 2002. An 419 anthropoid-specific locus of orphan C to U RNA-editing enzymes on chromosome 22. Genomics 420 79:285-296. 10.1006/geno.2002.6718

421 Koning FA, Newman EN, Kim EY, Kunstman KJ, Wolinsky SM, and Malim MH. 2009. Defining 422 APOBEC3 expression patterns in human tissues and hematopoietic cell subsets. $J$ Virol 83:9474423 9485. 10.1128/JVI.01089-09

424 Kouno T, Silvas TV, Hilbert BJ, Shandilya SMD, Bohn MF, Kelch BA, Royer WE, 425 Somasundaran M, Kurt Yilmaz N, Matsuo H, and Schiffer CA. 2017. Crystal structure of 426 APOBEC3A bound to single-stranded DNA reveals structural basis for cytidine deamination and 427 specificity. Nat Commun 8:15024. 10.1038/ncomms 15024 
428 Larijani M, Petrov AP, Kolenchenko O, Berru M, Krylov SN, and Martin A. 2007. AID associates

429 with single-stranded DNA with high affinity and a long complex half-life in a sequence-

430 independent manner. Mol Cell Biol 27:20-30. 10.1128/MCB.00824-06

431 Lehmann KA, and Bass BL. 1999. The importance of internal loops within RNA substrates of 432 ADAR1. J Mol Biol 291:1-13. 10.1006/jmbi.1999.2914

433 Lehmann KA, and Bass BL. 2000. Double-stranded RNA adenosine deaminases ADAR1 and 434 ADAR2 have overlapping specificities. Biochemistry 39:12875-12884.

435 Lorenz R, Bernhart SH, Honer Zu Siederdissen C, Tafer H, Flamm C, Stadler PF, and Hofacker 436 IL. 2011. ViennaRNA Package 2.0. Algorithms Mol Biol 6:26. 10.1186/1748-7188-6-26

437 Losey HC, Ruthenburg AJ, and Verdine GL. 2006. Crystal structure of Staphylococcus aureus 438 tRNA adenosine deaminase TadA in complex with RNA. Nat Struct Mol Biol 13:153-159. $439 \quad 10.1038 / \mathrm{nsmb} 1047$

440 Mitra M, Hercik K, Byeon IJ, Ahn J, Hill S, Hinchee-Rodriguez K, Singer D, Byeon CH,

441 Charlton LM, Nam G, Heidecker G, Gronenborn AM, and Levin JG. 2014. Structural 442 determinants of human APOBEC3A enzymatic and nucleic acid binding properties. Nucleic 443 Acids Res 42:1095-1110. 10.1093/nar/gkt945

444 Monajemi M, Woodworth CF, Benkaroun J, Grant M, and Larijani M. 2012. Emerging 445 complexities of APOBEC3G action on immunity and viral fitness during HIV infection and 446 treatment. Retrovirology 9:35. 10.1186/1742-4690-9-35

447 Nackley AG, Shabalina SA, Tchivileva IE, Satterfield K, Korchynskyi O, Makarov SS, Maixner 448 W, and Diatchenko L. 2006. Human catechol-O-methyltransferase haplotypes modulate protein 
449 expression by altering mRNA secondary structure. Science 314:1930-1933.

$450 \quad 10.1126 /$ science. 1131262

451 Namjou B, Gray-McGuire C, Sestak AL, Gilkeson GS, Jacob CO, Merrill JT, James JA,

452 Wakeland EK, Li QZ, Langefeld CD, Divers J, Ziegler J, Moser KL, Kelly JA, Kaufman KM,

453 and Harley JB. 2009. Evaluation of C1q genomic region in minority racial groups of lupus.

454 Genes Immun 10:517-524. 10.1038/gene.2009.33

455 Nishikura K. 2016. A-to-I editing of coding and non-coding RNAs by ADARs. Nat Rev Mol Cell 456 Biol 17:83-96. 10.1038/nrm.2015.4

457 Peng G, Greenwell-Wild T, Nares S, Jin W, Lei KJ, Rangel ZG, Munson PJ, and Wahl SM. 2007.

458 Myeloid differentiation and susceptibility to HIV-1 are linked to APOBEC3 expression. Blood 459 110:393-400. 10.1182/blood-2006-10-051763

460 Prohaska KM, Bennett RP, Salter JD, and Smith HC. 2014. The multifaceted roles of RNA

461 binding in APOBEC cytidine deaminase functions. Wiley Interdiscip Rev RNA 5:493-508.

$462 \quad 10.1002 /$ wrna.1226

463 Radanova M, Vasilev V, Dimitrov T, Deliyska B, Ikonomov V, and Ivanova D. 2015. Association 464 of rs172378 C1q gene cluster polymorphism with lupus nephritis in Bulgarian patients. Lupus 465 24:280-289. 10.1177/0961203314555173

466 Refsland EW, and Harris RS. 2013. The APOBEC3 family of retroelement restriction factors. 467 Curr Top Microbiol Immunol 371:1-27. 10.1007/978-3-642-37765-5_1

468 Sharma S, Patnaik SK, Kemer Z, and Baysal BE. 2017. Transient overexpression of exogenous

469 APOBEC3A causes C-to-U RNA editing of thousands of genes. RNA Biol 14:603-610.

470

$10.1080 / 15476286.2016 .1184387$ 
471 Sharma S, Patnaik SK, Taggart RT, and Baysal BE. 2016. The double-domain cytidine deaminase

472 APOBEC3G is a cellular site-specific RNA editing enzyme. Sci Rep 6:39100. 10.1038/srep39100

473 Sharma S, Patnaik SK, Taggart RT, Kannisto ED, Enriquez SM, Gollnick P, and Baysal BE. 2015.

474 APOBEC3A cytidine deaminase induces RNA editing in monocytes and macrophages. Nat

475 Commun 6:6881. 10.1038/ncomms7881

476 Shinohara M, Io K, Shindo K, Matsui M, Sakamoto T, Tada K, Kobayashi M, Kadowaki N, and

477 Takaori-Kondo A. 2012. APOBEC3B can impair genomic stability by inducing base substitutions

478 in genomic DNA in human cells. Sci Rep 2:806. 10.1038/srep00806

479 Wolf J, Gerber AP, and Keller W. 2002. tadA, an essential tRNA-specific adenosine deaminase

480 from Escherichia coli. EMBO J 21:3841-3851. 10.1093/emboj/cdf362

481 Zuker M. 2003. Mfold web server for nucleic acid folding and hybridization prediction. Nucleic 482 Acids Res 31:3406-3415.

FIGURE LEGEND

Figure 1. A3A and A3G prefer predicted stem-loop structures in their RNA substrates. (A)

486 A3A-mediated RNA editing in normoxia (N) and hypoxia and IFN-1 (HI) treated MEPs of three

487 independent donors. $\mathrm{C}>\mathrm{T}(\mathrm{U})$ editing is characterized by the emergence of a secondary $\mathrm{T}$ peak

488 (red) accompanied by a reduction in height of $\mathrm{C}$ peak (blue). $\mathrm{A}>\mathrm{G}$ silent nucleotide

489 polymorphism (SNP rs 172378) in C1QA RNA of donor 1 increases $\mathrm{C}>\mathrm{U}$ editing level (left) as an

490 additional base pair (represented by a dashed line) is predicted to form in the stem of the putative

491 stem-loop (right). Edited C is underlined. (B) A3A-mediated editing in WT and mutant SDHB

492 RNA. WT $S D H B$ RNA forms a putative tetra-loop flanked by a 5 bp stem. Mutations (M) are 
493 described above the stem-loop and the mutated nucleotides are colored red in the figure. The 494 average percentage RNA editing of $n=3(n=2$ for M1, 6 and 7$)$ is shown in bold and the standard 495 deviations are within parenthesis. The percentage RNA editing in c.136C $>\mathrm{U}$ was calculated using 496 allele-specific RT-qPCR (see methods), except M2, M8, 9 and 10 which were calculated using 497 the Sequencher ${ }^{\mathrm{TM}} 5.0$ software (see methods). WT RNA editing was set to $100 \%$ and the mutants 498 were calculated as a fraction of the WT. (C) A3A-mediated editing in WT and mutant APP (left) 499 and TMEM109 RNAs (right). WT APP RNA forms a putative tetra-loop flanked by a 5 bp stem. 500 WT TMEM109 forms a putative tetra-loop flanked by a $5 \mathrm{bp}$ stem and the unpaired adenosine (A) 501 bulges out. (D) A3G-mediated RNA editing of PRPSAP2 RNA, which forms a putative tetra-loop 502 flanked by a 4 bp stem. For (C) and (D), mutations (M) are described above the stem loop and the 503 mutated/inserted nucleotides are marked in red. The average percentage RNA editing of $n=3$ is 504 shown in bold and the standard deviations are within parenthesis. The percentage RNA editing 505 was calculated using the Sequencher ${ }^{\mathrm{TM}} 5.0$ software. ND: RNA editing not detectable (below 506 threshold). 


\section{Figure 1}

A3A and A3G prefer predicted stem-loop structures in their RNA substrates.

(A) A3A-mediated RNA editing in normoxia (N) and hypoxia and IFN-1 (HI) treated MEPs of three independent donors. $\mathrm{C}>\mathrm{T}(\mathrm{U})$ editing is characterized by the emergence of a secondary T peak (red) accompanied by a reduction in height of $C$ peak (blue). $A>G$ silent nucleotide polymorphism (SNP rs172378) in C1QA RNA of donor 1 increases C>U editing level (left) as an additional base pair (represented by a dashed line) is predicted to form in the stem of the putative stem-loop (right). Edited C is underlined. (B) A3A-mediated editing in WT and mutant SDHB RNA. WT SDHB RNA forms a putative tetra-loop flanked by a $5 \mathrm{bp}$ stem. Mutations (M) are described above the stem-loop and the mutated nucleotides are colored red in the figure. The average percentage RNA editing of $n=3$ ( $n=2$ for M1, 6 and 7) is shown in bold and the standard deviations are within parenthesis. The percentage RNA editing in c.136C $>U$ was calculated using allele-specific RT-qPCR (see methods), except M8, 9 and 10 which were calculated using the Sequencher ${ }^{\mathrm{TM}} 5.0$ software (see methods). WT RNA editing was set to $100 \%$ and the mutants were calculated as a fraction of the WT. (C) A3Amediated editing in WT and mutant APP (left) and TMEM109 RNAs (right). WT APP RNA forms a putative tetra-loop flanked by a $5 \mathrm{bp}$ stem. WT TMEM109 forms a putative tetra-loop flanked by a 5 bp stem and the unpaired adenosine (A) bulges out. (D) A3G-mediated RNA editing of PRPSAP2 RNA, which forms a putative tetra-loop flanked by a 4 bp stem. For (C) and (D), mutations (M) are described above the stem loop and the mutated/inserted nucleotides are marked in red. The average percentage RNA editing of $n=3$ is shown in bold and the standard deviations are within parenthesis. The percentage RNA editing was calculated using the Sequencher ${ }^{\mathrm{TM}} 5.0$ software. ND: RNA editing not detectable (below threshold). 
A

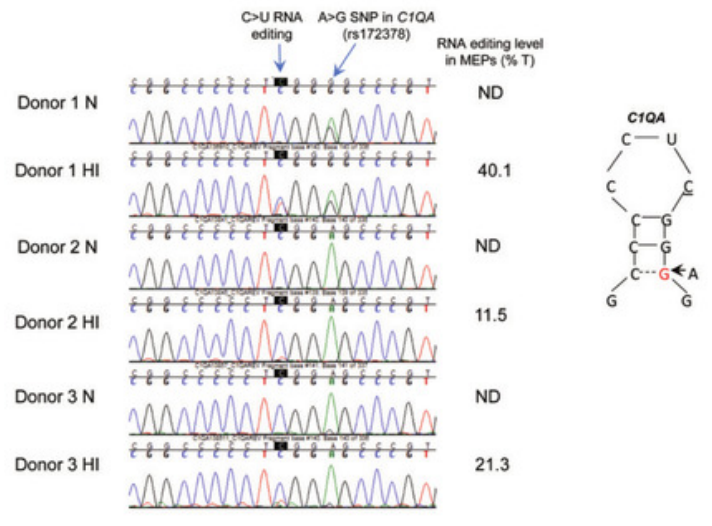

c

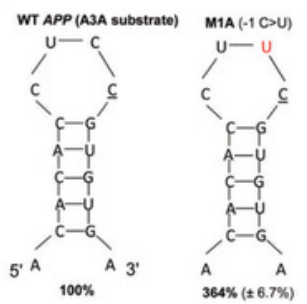

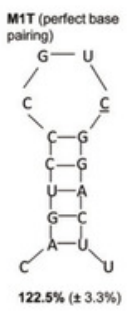

B

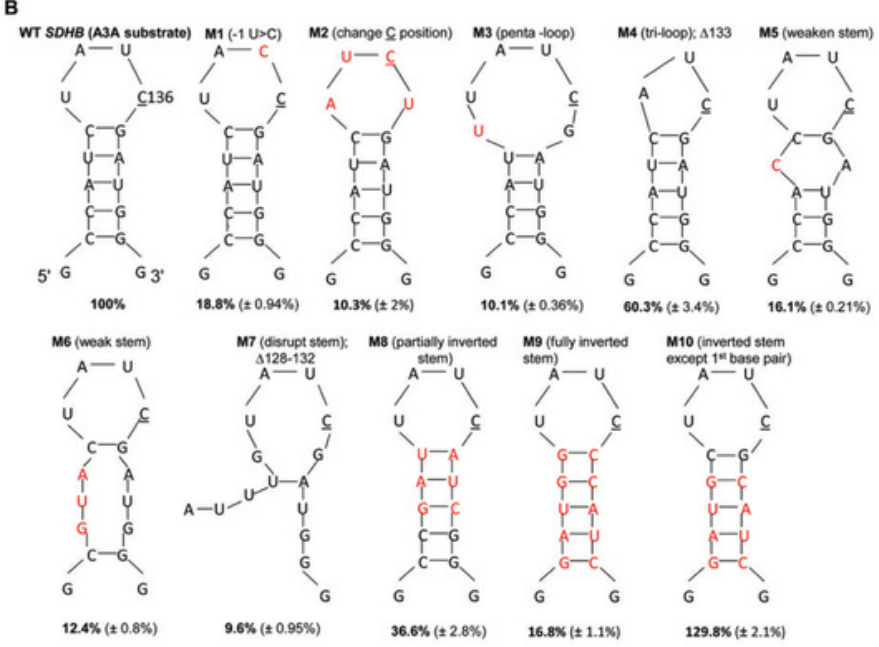

D
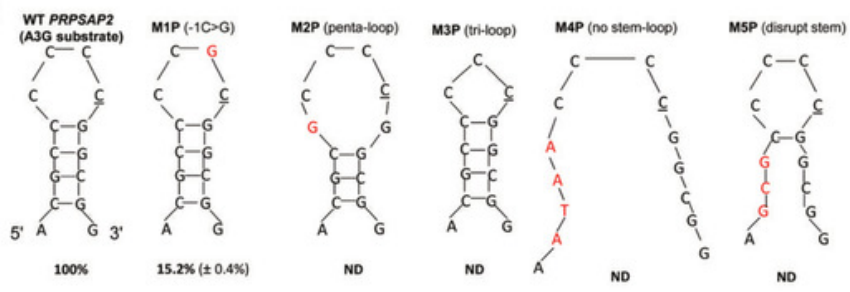

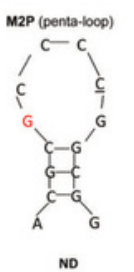

No

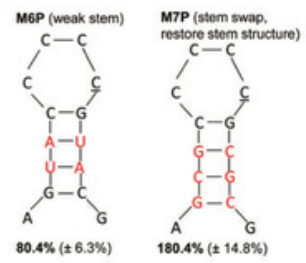

